\title{
Percutaneous coronary intervention and drug-eluting stents
}

13 See related articles pages 345, 361

Tnterventional cardiology 1 made its debut 27 years ago, when Andreas Grüntzig performed the first percutaneous transluminal coronary angioplasty in a human. Since then, rapid expansion of our knowledge of vascular pathology and its response to therapy, combined with tremendous advances in medical technology, has proif pelled this once obscure discipline to the forefront of treatment of symptomatic coronary artery disease. In the United States the number of percutaneous coronary interventions performed each year is nearly double that of coronary artery bypass surgery. And that is just the beginning. With the recent advent of drug-eluting stents, we are at the dawn of a new era of interventional cardiology. For the first time we are able not only to treat the mechanical consequences of coronary artery disease but also to control the pathobiological response of the vessel wall.

The artery wall consists of 3 layers: the intima, the media and the adventitia. Coronary artery disease is related to luminal narrowing or stenosis from atherosclerotic plaque buildup in the subintimal space, between the intima and the media. Over the past few decades the use of drug therapy with antiplatelet agents, anticoagulants and thrombolytic agents has improved clinical outcomes of patients with coronary artery disease; however, mechanical dilation of the stenotic arterial lumen remains the mainstay for the percutaneous treatment of the disease. First achieved with balloon angioplasty, mechanical dilation was later complemented with the insertion of bare-metal stents and now drug-eluting stents.

The principle of balloon angioplasty is simple: increase the size of the lumen at a stenosed area of a blood vessel to restore

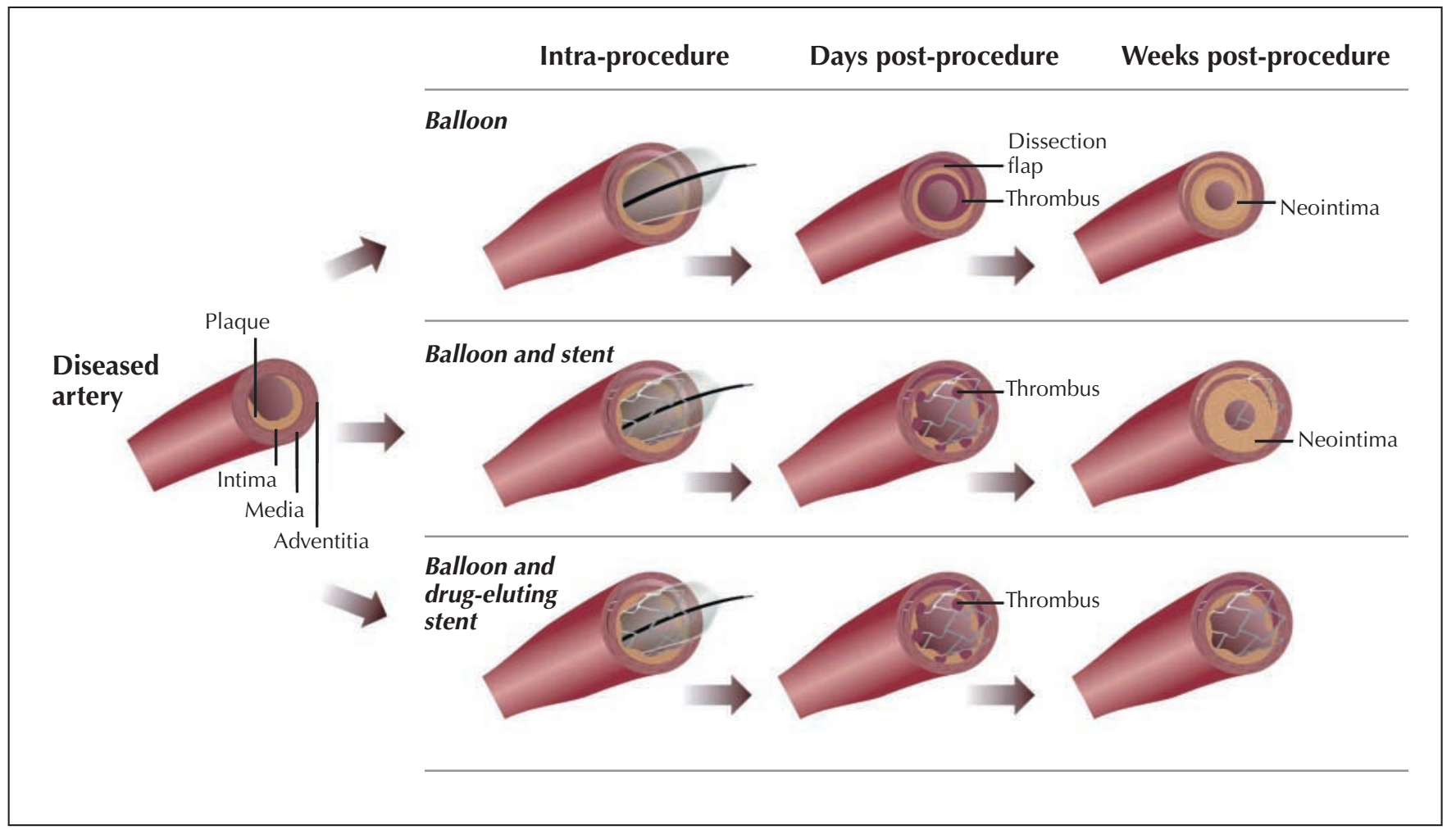

Vascular response to balloon angioplasty (top panel), balloon angioplasty plus bare-metal stent (middle panel) and balloon angioplasty plus drug-eluting stent (bottom panel). Top panel: Balloon inflation increases lumen diameter by stretching the media and the adventitia and causes plaque fracture and dissection; the lumen area decreases within days because of elastic recoil, and a thin layer of thrombus covers the lumen and fills the dissection plane; neointimal growth leads to further lumen loss weeks to months after the procedure. Middle panel: A bare-metal stent prevents elastic recoil and seals the dissection flap but causes deeper tissue injury and extensive thrombus formation around the stent struts; exaggerated neointimal formation caused by the stent negates the benefit of its scaffolding effect and results in in-stent restenosis. Bottom panel: A drug-eluting stent not only provides scaffolding to prevent elastic recoil, but the drug contained in the polymer coating of the stent inhibits neointimal proliferation. 
normal blood flow. This increase in arterial lumen size is achieved primarily by stretching the media and the adventitia. ${ }^{1}$

However, angioplasty itself is fraught with complications. Denudation of the endothelium and compression and fracturing of the atherosclerotic plaque also occur, which lead to multiple dissection planes in the subintimal and, sometimes, the medial space. The immediate vascular response to balloonrelated injury involves elastic recoil of the media and the adventitia toward their original calibers and the formation of a thin layer of thrombus lining the arterial lumen and filling the dissection planes. Subsequent recruitment of inflammatory cells, migration and proliferation of vascular smooth muscle cells, and deposition of extracellular matrix at the injury site lead to the formation of neointima. It was estimated that $70 \%$ of the gain in lumen size from balloon inflation was lost to elastic recoil shortly after angioplasty. Furthermore, the formation of dissection planes potentially gives rise to endothelial flaps that incite thrombus formation and acute obstruction of blood flow - a common complication at the time of or shortly after the procedure.

Metal stents were developed to overcome the limitations of balloon angioplasty, the rationale being that, by implanting a stent after balloon inflation, one could compress the dissection flap and prevent acute thrombus formation and at the same time avoid elastic recoil by scaffolding the artery and maintaining the gain in lumen area. However, compared with balloon angioplasty, stents can cause deeper vascular injury, more extensive thrombus formation and subsequently a greater degree of neointimal formation called neointimal hyperplasia. Neointimal growth into the stent lumen accounts for the majority of cases of late restenosis of the vessel. ${ }^{2}$ Proliferation and migration of vascular smooth muscle cells from the media into the intima is the key component of this maladaptive response. After stent deployment, platelets and leukocytes recruited to the injury site release growth factors and cytokines that stimulate division and propagation of vascular smooth muscle cells. At the same time, reendothelialization of the denuded arterial lumen occurs. Once the endothelium underlying the stent has been completely restored, only then is neointimal formation halted. The whole process generally takes several months, and clinical presentation of in-stent restenosis, typically in the form of angina, usually happens in $10 \%-50 \%$ of the cases within 6 months after the procedure. The rate of restenosis is higher among patients with long lesions, those with small-caliber vessels and patients with diabetes, especially if they require insulin treatment.

In the early 1990s sirolimus, a macrolide antibiotic with immunosuppressive and antimitotic properties, was found to be a potent inhibitor of vascular smooth muscle cell proliferation and migration. The drug interacts with mTOR receptors, causing downregulation of cyclin-dependent kinases and thus arresting cell growth. Systemic sirolimus therapy was used in renal transplant patients and was associated with significant side effects such as hyperlipidemia and thrombocytopenia. Local delivery of the drug was achieved by using stents coated with 2 polymers, the inner layer a polymer containing low doses of sirolimus and the outer layer a drug-free polymer acting as a barrier or controller for extended drug release. Several prospective randomized trials have shown profound inhibition of neointimal hyperplasia with sirolimuseluting stents. In a pivotal trial, ${ }^{3}$ 1058 patients were randomly assigned to receive either a baremetal or a sirolimus-eluting stent. Compared with the baremetal stents, the sirolimuseluting ones reduced the incidence of in-stent restenosis from $35.4 \%$ to $3.2 \%$. Despite a small drop in drug effect at the edges of the stent, which resulted in a higher incidence of restenosis at these sites than at sites inside the stent, there was still a dramatic $77 \%$ reduction in the need of repeat revascularization because of restenosis, from $22.3 \%$ to $6.9 \%$. In addition, sirolimus altered the pattern of in-stent restenosis from diffuse to focal, which made it easier to treat the restenotic lesion. A subgroup analysis showed that all patients at high risk for restenosis, including diabetic patients in general, benefited significantly from the sirolimus; however, a subsequent substudy revealed that the effect of the drug was much less dramatic in diabetic patients requiring insulin treatment. ${ }^{4}$

Parallel to the development of the sirolimus-eluting stent, another antiproliferative agent, paclitaxel, was used on a polymercoated stent platform. Paclitaxel promotes the assembly of highly stable microtubules. The stabilization of microtubules prevents cellular division. In the TAXUS IV trial, ${ }^{5} 1314$ patients were randomly assigned to receive either a bare-metal or a paclitaxel-coated stent. Again, there was a significant reduction in the rate of instent restenosis, from $26.6 \%$ to $7.9 \%$. This translated into a remarkable decrease in the need for ischemia-driven revascularization, from $12 \%$ to $4.7 \%$. Subgroup analysis showed that all of the patients at high risk for restenosis benefited from the paclitaxel just as much as the lowrisk patients. In other words, previously recognized high-risk determinants of restenosis, including insulin-requiring diabetes, seem to be neutralized with the use of paclitaxel-coated stents.

Drug-eluting stents are dramatically changing our approach to the treatment of coronary artery disease. For instance, patients with multivessel disease have traditionally been referred for coronary artery bypass surgery in the past. However, given the dramatic reduction in the rate of restenosis with drugeluting stents and the lower rates of target vessel failure with this treatment than with saphenous 
vein grafting, revascularization with drug-eluting stents is quickly becoming the preferred treatment for these patients.

In the future, drug-eluting stents may be used for the prevention of acute coronary syndrome. It is believed that acute coronary syndrome is caused by unstable plaques of mild to moderate stenosis $(<50 \%)$ that do not result in angina symptoms. Given the high event-free survival rate achieved with drug-eluting stents, one could argue for treating these lesions with stent therapy and potentially changing their natural history. In addition, the effect of drug-eluting stents on other difficult lesion subsets, such as left main coronary artery disease, bifurcation disease and chronic total occlusions, is currently under investigation.

However, it is important to keep in mind that the efficacy of the drug-eluting stent is based on avoiding recurrent angina and ad- ditional revascularization procedures. These stents do not reduce the incidence of myocardial infarction or death. From an economic perspective, a recent analysis of the SIRIUS trial showed that, even though the use of drugeluting stents significantly increased the immediate procedural cost, there was no significant difference in cost at 1-year follow-up because of the reduced need for repeat revascularization.

Over the past 27 years percutaneous coronary intervention has undergone changes at a breathtaking pace. With the recent development of the drug-eluting stent, an even more spectacular evolution is taking place that will forever change the landscape of interventional cardiology.

\section{Yi-ming Yang}

Lenox Hill Hospital

Issam Moussa

Columbia University Medical Center Cardiovascular Research Foundation New York, NY

\section{References}

1. Mintz GS, Popma JJ, Pichard AD Kent KM, Satler LF, Wong SC, et al. Arterial remodeling after coronary angioplasty: a serial intravascular ultrasound study. Circulation 1996;94:35-43.

2. Hoffmann R, Mintz GS, Dussaillant GR, Popma JJ, Pichard AD, Satler LF, et al. Patterns and mechanisms of in-stent restenosis: a serial intravascular ultrasound study. Circulation 1996; 94:1247-54.

3. Moses JW, Leon MB, Popma JJ, Fitzgerald PJ, Holmes DR, O'Shaughnessy C, et al; SIRIUS Investigators. Sirolimus-eluting stents versus standard stents in patients with stenosis in a native coronary artery. $N$ Engl $7 \mathrm{Med}$ 2003;349(14):1315-23.

4. Moussa I, Leon MB, Baim DS, O'Neill WW, Popma JJ, Buchbinder $\mathrm{M}$, et al. Impact of sirolimus-eluting stents on outcome in diabetic patients: a SIRIUS (SIRolImUS-coated Bx Velocity balloon-expandable stent in the treatment of patients with de novo coronary artery lesions) substudy. Circulation 2004;109:2273-8.

5. Stone GW, Ellis SG, Cox DA, Hermiller J, O'Shaughnessy C, Mann JT, et al; TAXUS-IV Investigators. A polymer-based, paclitaxel-eluting stent in patients with coronary artery disease. N Engl 7 Med 2004;350(3): 221-31

\section{SCience ANd Medicine}

\section{Do stem cells cause gastric cancer?}

A lthough the link between Helicobacter pylori infection and gastric cancer is well established, new research suggests that stem cells play an important role in the development of this malignant disease. JeanMarie Houghton and colleagues recently showed that $H$. pylori-induced inflammation in mice caused the migration of stem cells originating from bone marrow to the stomach, where they subsequently developed into gastric tumours. ${ }^{1}$ Previous evidence suggests that bone-marrowderived cells have a reparative function on being recruited to areas of injury or inflammation. $\approx$ The idea that these cells might ठิ also play a role in the develop. ment of cancer revisits a concept 丞 that arose partly from the observation in the 1970 s that only $1 \%$ - of leukemia cells grow into colonies in vitro, an ability that Olater earned these cells the label "cancer stem cells." Houghton and colleagues' research suggests that similar stem cells may give rise to gastric cancer, a finding that presents a new way of think-

ing about the pathogenesis of a disease that is the second leading cause of cancer-related deaths worldwide, killing nearly 600000 people each year.

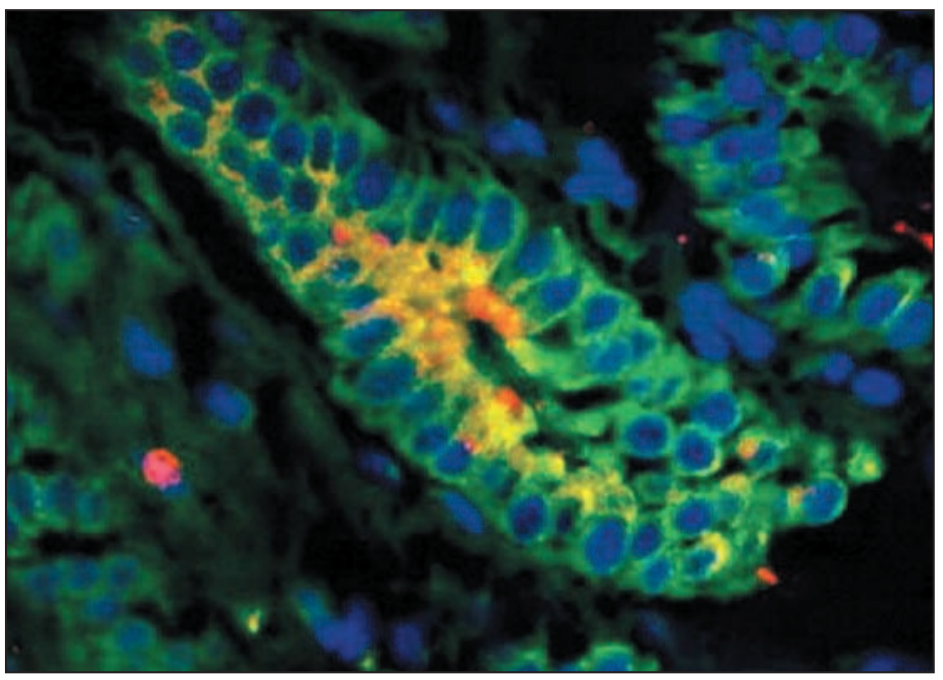

Bone-marrow-derived stem cell that has differentiated into a gastric epithelial cell. Reprinted, with permission, from Houghton et al. ${ }^{1}$ (C) 2004 American Association for the Advancement of Science. 